\title{
La chefferie coutumière face au projet de décentralisation dans une localité de l'Ouest nigérien
}

\author{
Eric Komlavi Hahonou
}

\author{
(2) OpenEdition \\ Journals \\ Édition électronique \\ URL : http://journals.openedition.org/apad/141 \\ DOI : 10.4000/apad.141 \\ ISSN : 1950-6929 \\ Éditeur \\ LIT Verlag \\ Édition imprimée \\ Date de publication : 1 décembre 2002 \\ Référence électronique \\ Eric Komlavi Hahonou, «La chefferie coutumière face au projet de décentralisation dans une localité \\ de l'Ouest nigérien », Bulletin de l'APAD [En ligne], 23-24 | 2002, mis en ligne le 15 décembre 2006, \\ consulté le 07 septembre 2020. URL : http://journals.openedition.org/apad/141 ; DOI : https://doi.org/ \\ 10.4000/apad.141
}

Ce document a été généré automatiquement le 7 septembre 2020.

Bulletin de I'APAD 


\title{
La chefferie coutumière face au projet de décentralisation dans une localité de l'Ouest nigérien
}

\author{
Eric Komlavi Hahonou
}

1 A l'instar de quelques pays voisins de l'Afrique de l'Ouest francophone, au Niger la décentralisation est un projet relativement ancien. Relancée par le discours de La Baule, et malgré les aléas de la démocratie au Niger (Conférence Nationale, rébellion armée, élections présidentielles de 1993, coup d'état de 1996, élections locales de février 1999, annulation des élections, nouveau coup d'état en avril 1999, retour à la démocratie en décembre 1999), la mise en œuvre de la décentralisation reste à l'état de projet de réforme. Le cadre législatif de cette réforme administrative, maintes foi remanié, propose la communalisation intégrale de tous les chefs-lieux de canton (fief de la chefferie coutumière sédentaire) ainsi que de certains groupements (nomades). Il s'agit, d'une part, de transférer les pouvoirs de décisions de l'Etat central vers le niveau local, mais d'autre part, et surtout, la réforme bouleverse profondément les modes de gestion actuels et les prérogatives des actuels tenants du pouvoir au niveau des quartiers, villages, cantons... en procédant à l'élection d'un conseil municipal et d'un maire.

2 La "bonne gouvernance" au Niger est, comme partout, avancée pour justifier la réforme. Je comprendrai ici le concept de gouvernance assez classiquement comme la manière dont le pouvoir est exercé dans la gestion, la résolution éventuellement, de problèmes qui se posent à une communauté, une collectivité, pouvoir exercé par des acteurs étatiques ou non. A travers ce concept, il est permis d'étudier les rapports entre les tenants du pouvoir et les gouvernés, et plus loin d'appréhender la gestion des biens publics par les multiples acteurs au niveau de différentes arènes locales. C'est à ce niveau (local level politics), celui du "village réel", entité sociale et politique, appréhendé comme "champ semi-autonome" (selon Sally Moore 1978) ou "arène" (Bailey, 1969), que je me propose d'aborder la gouvernance comme une confrontation des logiques d'acteurs autour d'enjeux locaux, plutôt que généraux ou globaux. A ce niveau de 
lecture des micro-enjeux peuvent apparaître et seule une étude des réalités de la décentralisation "au ras-du-sol" va permettre d'observer le changement politique et social dans toute sa diversité et sa complexité.

3 C'est à l'arène politique locale de Bankilaré dans l'extrême Ouest nigérien que je m'intéresserai ici. Le choix du village de Bankilaré comme site de recherche est justifié par son statut particulier : selon les lois actuellement en vigueur (loi n ${ }^{\circ} 98-029$ du 14 septembre 1998) cette localité se présente comme une future "commune rurale sans territoire".

Ce Poste Administratif (PA) fut créé en 1959 pour administrer uniquement les populations nomades dispersées dans les différents cantons songhay de la zone et relevant des chefferies de groupement peul et kel tamasheq ${ }^{1}$, Dans le contexte de la mise en place de la décentralisation, cette caractéristique paradoxale en fait l'arène politique locale la plus emblématique au Niger. Depuis 1996, tous les textes de lois sur la décentralisation y font référence comme une exception, un cas particulier.

On considèrera cette arène non pas comme un village fonctionnant en vase clos, mais en le situant dans un contexte plus large qui permet d'envisager les liens socio-politiques de cette arène avec les autres arènes locales et avec l'arène nationale ${ }^{2}$.

Une tentative de catégorisation des groupes stratégiques ${ }^{3}$ de l'arène politique locale au Niger permet de dégager six catégories de pouvoirs locaux : les pouvoirs coutumiers (la chefferie traditionnelle), les pouvoirs administratifs (agents des services administratifs et techniques déconcentrés), les pouvoirs religieux (marabouts, prêtres guérisseurs...), les pouvoirs politiques (partis), les pouvoirs économiques (commerçants, gros éleveurs...) et les pouvoirs associatifs (association de ressortissants, associations et comités divers liés aux projets de développement).

7 La décentralisation offre l'opportunité d'un changement social, d'une redistribution des pouvoirs au niveau local, et fait, à ce titre, l'objet de stratégies anticipatives de la part des différents acteurs (anciens et nouveaux) qui se positionnent dans l'arène politique locale. Elle porte potentiellement en elle des perspectives de renforcement de phénomènes contestataires en ouvrant la concurrence pour l'accès au pouvoir au niveau local. De rapports d'autorité et de hiérarchie (chefs et sujets, maîtres et esclaves ${ }^{4}$, hommes et femmes...) c'est à des rapports égalitaires que prétend aboutir la décentralisation impulsée par l'Etat. Aussi, les risques sont-ils plus particulièrement perçus par les actuels tenants du pouvoir local, en particulier les chefs coutumiers et religieux, qui disposent toutefois encore de marges de manœuvre face à un projet de décentralisation, imposé par le haut, aux limites encore floues pour la plupart des acteurs d'en bas.

8 Dans un premier temps je retracerai le cadre géographique dans lequel évoluent les acteurs locaux, en mettant l'accent sur les dynamiques de changement en cours dans les rapports sociaux, les relations à l'espace et les modes de production, On se focalisera ensuite sur la future commune rurale de Bankilaré pour y examiner la place et le rôle des différents acteurs stratégiques qui participent à la gouvernance locale actuelle des affaires des tribus ou des confédérations, Le rôle, le positionnement et le point de vue des chefs traditionnels, déjà identifiés comme grands perdants de la décentralisation, sera privilégié dans la présente analyse. Enfin, je préciserai les enjeux affichés et les enjeux cachés (enjeux de pouvoirs, enjeux fonciers et enjeux identitaires) de la "dispute" pour la commune et tenterai de montrer qu'ici les perspectives de mise en œuvre d'une décentralisation imposée aux acteurs locaux, loin de conduire à une 
gestion démocratique et participative des affaires locales (argument mis en avant dans l'exposé des motifs des projets de loi successifs portant création des communes...) semblent plutôt augurer le renforcement des pouvoirs en place.

Cadre géographique et humain

9 Bankilaré est un petit bourg de quelques milliers d'habitants, essentiellement kel tamasheq, situé dans le canton de Gorouol, dans l'extrême Nord-Ouest de l'arrondissement de Téra aux frontières du Mali et du Burkina Faso. Cette zone, autrefois décrite comme riche, était réputée comme grenier à mil de l'arrondissement (jusqu'en 1970) et possédait d'importantes potentialités pastorales ainsi que des cultures de rente (niébé de décrue). Elle est considérée aujourd'hui comme une zone relativement ingrate aux sols pauvres, A la faible fertilité des sols et la rareté des terres cultivables (un peu plus de $900 \mathrm{~km} 2$ seulement sur une superficie totale de $4.480 \mathrm{~km} 2$ ) s'ajoutent des contraintes climatiques fortes qui réduisent souvent à néant les efforts des cultivateurs.

10 Les meilleures terres sont situées autour du lit de la rivière Gorouol (dont le canton tire son nom) et ses affluents (Ouanégori) où sont regroupés la majorité des 27 villages songhay que compte le canton. Les terres inondées des abords du Gorouol permettent la pratique des cultures de décrue (niébé), cultures également praticables autour de quelques mares semi-permanentes. Les revenus de ces cultures et ceux liés à l'émigration vers les pays côtiers (du Nigeria à la Côte d'Ivoire) ont permis à de nombreux cultivateurs de réinvestir dans le petit élevage qui le plus souvent constitue une forme d'épargne et une activité secondaire.

11 L'espace restant (zone des dunes, zone des mares et nord de la vallée du Gorouol) est plus favorable à l'élevage extensif des bovins et des petits ruminants, élevage qui était autrefois l'apanage de pasteurs nomades kel tamasheq et peuls. Sous l'action conjuguée des grandes sécheresses (la grande famine de 1911-1913 est généralement citée comme une date de référence) et d'une politique coloniale de sédentarisation des nomades, les iklan. anciens captifs, libérés du joug de leurs maîtres imajeghen (nobles), ont intensifié leurs pratiques agricoles (cultures céréalières pluviales), sans toutefois en faire une activité principale. Cette conversion tardive n'est pas sans incidence sur les rapports que ces anciens pasteurs nomades entretiennent vis-à-vis du foncier (originellement caractérisés par une absence de recherche d'appropriation foncière, puis progressivement des pratiques de défrichage pour l'extension des terres de culture, et des pratiques d'emprunt de terres aux songhay) et sur les représentations que leurs voisins sédentaires songhay s'en font. Néanmoins, la sédentarisation aidant, les anciens nomades ont peu à peu revendiqué les terres cultivées, revendications causant souvent des litiges voire des conflits.

12 Par ailleurs, les passages des troupeaux sont aussi l'objet de conflits récurrents entre pasteurs et cultivateurs, malgré l'existence de nombreux couloirs de passage (parfois abusivement mis en culture) qui permettent théoriquement aux troupeaux de transiter des terres agricoles du Sud vers les terres pastorales du Nord (Sud Mali et Nord-Est du Burkina Faso). La récolte du niébé (le haricot d'une part et les fanes par la suite) est souvent tardive et le passage d'un grand troupeau dans un champ non récolté occasionne de gros dégâts (parfois volontairement causés par les bergers) pour les cultivateurs. Aussi, comme dans de nombreuses régions à vocation agropastorale, les conflits sur les dégâts champêtres sont fréquents et il n'est pas rare que les altercations 
dégénèrent violemment, surtout s'il s'agit de litiges opposant des individus d'ethnies différentes ${ }^{5}$.

Bankilaré : arène politique locale "à part"

13 Le Canton de Gorouol compte trois arènes politiques locales d'importance, deux d'entre elles sont les villages songhay de Yatakala et Kolman, respectivement ancien et nouveau chef-lieu du canton, situés au nord du canton de part et d'autre du Gorouol. La troisième arène politique locale est le village de Bankilaré, qui regroupe essentiellement des populations kel tamasheq de différentes tribus.

Bankilaré est un petit bourg du Canton de Gorouol, qui fut érigé en Poste Administratif (P.A.) en 1959, à la veille de l'Indépendance du Niger. En tant que P.A., Bankilaré est doté de quelques services déconcentrés de l'Etat. Parmi ces services, il faut signaler la présence d'une brigade de gendarmerie, des forces de sécurité sous la direction du Chef de Poste Administratif, un centre de santé, une école primaire (créée en 1956), et une panoplie d'agents de l'Etat sans bureaux chargés d'assurer les services d'Elevage, du Plan, de l'Agriculture et des Eaux et Forêts.

Le P.A. comme partout ailleurs au Niger remplit une fonction théorique de rapprochement de l'administration vers les administrés (état civil, gestion de la ville, règlement des litiges champêtres) mais en l'occurrence sa spécificité tient au fait que ce poste fut créé en 1959 pour administrer à part les populations des groupements kel tamasheq Tinguereguedech-Loghmatten et Doufrafrak et du groupement peul Gaobé. Du point de vue de la loi qui définit le découpage territorial, ces chefferies de groupement kel tamasheq se trouvent implantées sur un espace territorial qu'elles ne maîtrisent pas, celui du Canton du Gorouol. Les peul Gaobé, quant à eux, ne résidant pratiquement plus sur le territoire du Canton du Gorouol, sont évacués ${ }^{6}$ du paysage des acteurs locaux. Il reste que le décret qui créait le P.A. instituait du même coup un traitement séparé des Kel Tarnasheq et des Songhay. Ceci a occasionné un sentiment de frustration de ces derniers qui se plaignent d'avoir à se rendre à la Sous-Préfecture de Téra, située à quelques 120 kilomètres de leur chef-lieu de Canton (à l'époque Yatakala) au sud de l'arrondissement, pour toute formalité administrative ou tout recours aux services techniques.

16 Il faut ajouter à la particularité de Bankilaré son statut de circonscription spéciale. Les circonscriptions spéciales ont été mises en place au Niger pour assurer la représentation des groupes minoritaires. Giraut (1999) avance que ces circonscriptions spéciales constituent en quelque sorte une compensation à la cantonalisation pour des groupes minoritaires ou de statut servile, qui furent marginalisés lors de la reconnaissance coloniale d'autorités coutumières. Elles renforcent le caractère explicitement ethnique des localités concernées.

17 Depuis l'érection du village en P.A., les pouvoirs coutumiers et religieux ont dû composer avec l'administration, nouvel acteur devenu central dans l'arène politique locale. Les chefs de poste administratif, administrateur de la France d'Outre Mer puis "commandant indigène", ont du poursuivre leur mission de gestion des nomades par l'intermédiaire de la chefferie kel tamasheq, qui quoique affaiblie, apparaissait encore incontournable. Néanmoins pour celle-ci, cette mise sous tutelle rapprochée s'est traduite par une certaine restriction de ses libertés (en particulier les divers abus de pouvoir: extorsion de biens au titre de la dîme, rapt d'enfants en vue de leur asservissement, violences diverses, etc.). Une dimension importante du travail du chef de poste consistait à tenter d'arbitrer les nombreux conflits qui ne manquaient pas de 
naître entre les tenants de la chefferie, toujours promptes à récupérer ce qui jadis constituait un dû de leurs tributaires et dépendants, et ces derniers toujours plus avides des libertés que la colonisation leur avait octroyées au point de refuser le paiement de l'impôt. La gestion des plaintes diverses adressées directement à l'administration, le règlement des différends sur la propriété du bétail, l'impératif de contrôle des comportements d'incivisme fiscal... conduisirent le commandant de cercle de Téra à constituer un dossier sur les "Principes de commandement", mais surtout à ménager les chefs et leur statut, relais indispensables d'une administration qui eut de grandes difficultés à s'imposer aux nomades (archives de Téra, 1963).

L'avènement du multipartisme fut un autre tournant auquel la chefferie dut faire face dans la composition du pouvoir local. Mais là aussi ce furent les partis politiques qui, n'ayant pas véritablement d'assise locale, vinrent à elle pour tenter de gagner un levier électoral.

Organisation sociopolitique et gouvernance des groupements kel tamasheq de Bankilaré

19 Les deux groupements dont la représentation à Bankilaré est assurée par les Chefs de Groupement Tinguereguedech-Loghmatten et Doufrafrak sont de taille très inégale : le premier comprend 72 tribus (tawshiten) regroupant plus de 15000 personnes, tandis que le second n'en compte que 5, soit 2000 personnes environ. De fait, nous verrons que leur organisation politique est assez différente. Ils ont cependant en commun un système politique très hiérarchisé et centralisé, typique des aristocraties guerrières kel tamasheq, comprenant trois niveaux d'organisation du pouvoir (taghmar): un chef suprême qui est l' amenokal wan ettobel ou chef de groupement, secondé dans ces tâches par ses enfants et ses conseillers, parmi lesquels les marabouts (ineslemen) occupent une place prépondérante, et des chefs de tribus ou fractions (amenokal wan tawshit).

Différents statuts sociaux caractérisent le schéma d'organisation classique de la hiérarchie des kel tamasheq, avec à son sommet les aristocrates imajeghen, puis les hommes libres tributaires ou imghad, les artisans inadan et les captifs iklan à la base de cette pyramide. Le statut de chaque individu détermine généralement sa place dans les prises de décisions (Bourgeot 1995), les captifs au bas de la hiérarchie sociale ayant généralement le moins de poids. Toutefois, le statut d'inadan permet à certains d'occuper des positions sociales particulières qui leur permettent d'assumer des tâches de conseiller du chef ou de représentation notamment auprès des autorités administratives.

21 Il est à noter que ces éléments diffèrent d'un groupe à l'autre, notamment ici dans le cas du groupement Doufrafrak dont le schéma d'organisation est simplifié du fait que leur nombre est très restreint: "toutes les tribus qui restent pour mon groupement sont mes esclaves directs" (le chef de groupement, oct. 2001). Ces 5 tribus sont essentiellement le résultat d'un fractionnement (Doufrafrak I à IV). Le chef de groupement est lui-même peu présent dans son chef-lieu, préférant résider près des sphères de décision politiques et administratives de Téra et de Niamey. Les difficultés d'administration liées au dispersement des tribus sont levées par la désignation informelle par l'amenokal de délégués (au nombre de sept), qui s'ajoutent au lots des cinq chefs de tribus officiels pour gérer des populations relevant du groupement, à la différence près que le chef de groupement peut les révoquer à tout moment puisque l'administration publique ne les reconnaît pas. 

assemblage de groupes hétérogènes de populations de culture et de langue tamasheq qui, au hasard de leurs fuites et/ou conquêtes, par vagues successives, se sont retrouvés sur un même territoire sur la rive droite du fleuve Niger (appelée "Gunna") et placés sous l'autorité du chef des Tinguereguedech-Loghmatten. descendant des Oullimenden originaires de Ménaka (dans l'actuel Mali) qui au milieu du XVIII ème siècle se serait réfugié avec quelques fractions (Loghmatten, Ibahawan) de sa Confédération d'origine sur la rive "Gunna". Le petit groupe ainsi constitué nomadisa avec son cheptel, continuant les razzias et batailles, s'alliant tantôt aux uns tantôt aux autres, et parvint à dominer entièrement la rive Gunna jusqu'au moment de la pénétration coloniale française à laquelle ils opposèrent une farouche résistance.

De 54 fractions (ou tribus) dénombrées au recensement de 1954, les Tinguereguedech-Loghmatten ou Kel Igirer regroupent aujourd'hui 72 tribus. Cet accroissement est lié à un ensemble de facteurs convergents parmi lesquels il faut citer des phénomènes de scissions internes (fractions liées à des luttes pour le pouvoir de deux candidats à la succession d'un chef), à la recherche d'espaces de pâturage, à des stratégies d'éloignement des maîtres en vue d'une plus grande autonomie ${ }^{7} d^{\prime}$ 'une partie de la tribu ou de tribus vassales, ainsi que des mouvements d'intégration de nouvelles tribus en provenance du Mali voisin ou de la rive droite du fleuve (fuyant des disettes, des sécheresses ou des maitres trop exigeants). L'administration a également poussé à cette multiplication des fractions, dans la mesure où pour être Chef de groupement, il est en principe nécessaire de disposer de nombreuses tribus. Plus on possède de tribus sous sa coupe et mieux on est rémunéré ${ }^{8}$.

列 guerre imajeghen (nobles) descendants d'Ashwa, parmi lesquels un Conseil (aminiye) choisissait le candidat le plus apte à assumer les fonctions d'amenokal wan ettobel. Généralement le choix portait in jïne sur deux candidats, supportés chacun par des alliés au sein du Conseil. Il n'est pas rare que les choix opérés aient entraîné des dissensions ultérieures et des troubles dans la gestion de la Confédération consécutifs aux manigances du camp adverse. Le Conseil a également un rôle de contrôle et de sanction éventuelle du Chef de groupement. La destitution d'un Chef de groupement peut intervenir (théoriquement) dès lors que le Conseil estime qu'il ne s'acquitte pas correctement de ses missions. L'aminiye n'est pas une institution stable et matérialisée. Les membres de ce conseil sont des conseillers de l'amenokal, appelés à l'occasion pour se prononcer sur les affaires internes et externes de la Confédération.

attributions traditionnelles (pré-coloniales) du chef de guerre chez les Tinguereguedech ne diffèrent guère de celles des chefs des autres confédérations kel tamasheq : gestion des affaires extérieures, contrôle des mouvements de transhumance des groupes à l'occasion des cures salées, accueil des étrangers et gestion des demandes de protection de la part de tribus faisant scission d'avec leur confédération d'origine, contrôle des mouvements caravaniers, responsabilité des expéditions guerrières et des alliances, gestion des problèmes non résolus au niveau des chefs de tribus... Les pouvoirs et prérogatives des chefs ont bien entendu beaucoup régressé depuis la domination coloniale française. En 1936, puis en 1955, l'administration française établissait des textes réglementant les domaines de compétences des chefs, "responsables de la bonne administration" de leur collectivité, du maintien de l'ordre,... en tentant de concilier les droits coutumiers reconnus et les exigences d'une 
administration orientée par les priorités de paix sociale et de civisme fiscal. Bien que les textes désignent un unique responsable, dans les faits, qu'il s'agisse d'affaires internes ou externes à la Confédération, aucune décision n'est prise par l'amenokal wan ettobel seul. Pour la plupart des décisions courantes, le Chef de groupement consulte en premier lieu ses proches (vivant dans le même campement), qui sont ses propres enfants (auxquels il a délégué une bonne partie de ses tâches: collecte de l'impôt, conciliation et règlement de disputes et litiges mineurs...), sa femme et son "forgeron-ministre". Le cercle de ses conseillers s'élargit dès lors que les questions à traiter sont de plus grande importance (règlement de problèmes entre tribus, entre une tribu et un groupe extérieur ou tout problème concernant l'ensemble de la Confédération... tel que le projet de loi sur les communes), certains notables parmi les imajeghen, les Ibahawan (tribu d'anciens esclaves affranchis), les ineslemen (groupe paraboutique, ici représentés par les Kel Elsuk ${ }^{9}$ ) et les Loghmatten (tribu d'imghad guerriers) sont convoqués. Les motifs les plus récents de la réunion de cette assemblée des notables furent, à titre d'exemple, le choix du candidat à promouvoir pour le siège de député que leur accorde le statut de circonscription spéciale de Bankilaré, ou l'attitude à adopter face à la nouvelle de la suppression de la Commune de Bankilaré (sur laquelle nous reviendrons plus loin).

Tout de suite, il semble intéressant d'examiner la gouvernance au quotidien en se penchant sur le rôle d"'auxiliaire de l'administration" attribué au Chef de groupement, devenu courtier ${ }^{10}$ institutionnalisé du règlement des affaires nomades. Un bref rappel historique sur l'état des relations entre agents de l'Etat et administrés permettra de mieux saisir ce rôle de courtier qu'occupe la chefferie coutumière kel tamasheq aujourd'hui à Bankilaré.

Les chefs : courtiers institutionnalisés

Il est de fait assez significatif de constater que les populations désignées sous le terme Tinguereguedesh ne se reconnaissent pas dans ce vocable. La plupart des dénominations couramment utilisées aujourd'hui pour désigner les groupements, tribus et même des individus, ont été le fait de l'administration coloniale, à laquelle le mensonge était souvent opposé en guise de résistance à l'occupant. Le terme Tinguereguedech signifie "je suis sous la protection de", il désigne en fait le nom d'une tribu d'esclaves qui était située à proximité immédiate du campement de leurs maîtres, les Kel Igirer ${ }^{11}$.

De la même façon, le groupement Doufrafrak (litt. "derrière la clôture, le parc des animaux") revendique aujourd'hui le nom de Kel Ansongo, les Doufrafrak étant une tribu iklan (esclaves, captifs) de ces derniers.

Cette défiance à l'égard de l'occupant installé était partagée. La puissance coloniale ayant eu à faire face à plusieurs révoltes jusqu'en 1915-1916, se méfiait toujours de ces Kel Tamasheq et ces Peuls "turbulents et mobiles".

30 Sans approfondir les rapports ambigus, décrits par Bernus (1974), entre l'élite kel tamasheq et l'administration française, il faut rappeler qu'il est resté une défiance réciproque entre administrateurs et nomades jusqu'à nos jours, les agents de l'administration étant, en effet, souvent des "étrangers" (sédentaires hausa ou zarma le plus souvent).

31 L'accès à la justice fournit un bon exemple de la position stratégique occupée par la chefferie dans le règlement des affaires internes :

"Par exemple en cas de vol... nous dit le Commandant de Brigade de gendarmerie du Poste Administratif, la victime ne peut pas venir directement à la gendarmerie pour 
se plaindre. Il va aller voir son chef de tribu. L'autre l'envoie au chef de groupement. A chaque étape il faut qu'ils bouffent quelque chose. Il faut le thé et la cola de l'enfant du chef qui va saisir la gendarmerie... Comme ça les chefs de tribu et de groupement vont toucher. Leurs administrés sont des gens qui sont dans les tiroirs. Aucun nomade ne va venir directement au P.A, ou à la gendarmerie. On le fait toujours accompagner... L'intermédiaire, c'est le "guide". Lui, il est indispensable ! Le guide est de Bankilaré, il connaît l'administration même s'il n'a pas fait l'école. Les guides essentiels ce sont les enfants du chef. Ils n'ont pas de champ, ils n'ont rien d'autre qu'être les guides".

entourage de conseillers) contribuent à maintenir au plus bas le taux de scolarisation en freinant la création d'écoles (c'est le cas de l'école de Wisiwisi, village d'iklan, soutenue par une association de lutte contre l'esclavage. Le Chef de groupement a empêché sa création pendant près d'un an en refusant de signer le dossier à transmettre à l'inspection de l'enseignement primaire). La stratégie de tenir les sujets dans l'ignorance est aussi adoptée par les pouvoirs religieux qui, paradoxalement, n'enseignent pas le Coran à leurs sujets, de façon à éviter des revendications de leurs captifs fondées sur le droit musulman.

Ainsi, l'accès au service public se trouve entravé par les chefs traditionnels plutôt que facilité. Si entre extorsion et service rendu la limite peut sembler difficile à déterminer, il reste que les intérêts des uns et des autres peuvent paraître antagonistes. Pourtant les administrés sont aussi demandeurs vis-à-vis d'une chefferie censée leur apporter une protection contre l'Etat. C'est néanmoins au détriment des administrés, plus sujets que citoyens, que cette fonction d'intermédiation, qui participe directement à la gouvernance locale, se perpétue depuis la colonisation.

Bulletin de l'APAD, 23-24 | 2002 

l'acheminement et la distribution de l'aide alimentaire (systématiquement détournée) ou dans l'implantation des projets de développement, il suffira ici de souligner que les chefs coutumiers occupent dans les arènes locales des places centrales et des positions de pouvoir hégémonique qu'ils n'entendent pas perdre, malgré la redistribution de la donne politique que tente de mettre en œuvre l'Etat et ses partenaires du Nord à travers la décentralisation.

Le projet de décentralisation et le cas Bankilaré

Selon les lois de 1996, qui constituent la base fondatrice de la réforme nigérienne, les régions, départements et communes sont érigés en collectivités territoriales. Les arrondissements, sont en revanche des circonscriptions administratives d'encadrement des communes. Les structures coutumières participent de l'organisation administrative des circonscriptions territoriales et gardent leur statut de "courroie de transmission" de l'administration. Ce schéma global satisfaisait les exigences des leaders de la "Rébellion touarègue" (accord de paix de 1995) - rébellion à laquelle les Kel Tamasheq du Ourma ne participèrent pas ${ }^{12}$ qui exigeaient une plus grande autonomie dans l'espace et la gestion de leur avenir ${ }^{13}$.

Les lois de 1998 viennent compléter celles de 1996. La loi 98-29 porte création des communes et fixe leurs limites. Au niveau communal, qui nous intéresse plus particulièrement ici, il était envisagé de conserver les 21 communes urbaines existantes, de transformer les 24 chefs-lieux d'arrondissement en autant de communes urbaines et enfin d'ériger les 27 postes administratifs en 27 communes rurales. Le nombre total de communes est de $73^{14}$. C'est le schéma préconisé par le Haut Commissariat à la Réforme Administrative et à la Décentralisation (HCRA/D) et retenu par le gouvernement de la IV ème République du Niger pour les élections de février 1999. Le HCRA/D signalait avec insistance les "risques de résurgence de féodalités locales, d'aggravation de déséquilibres régionaux, de mauvaise gestion, d'atteinte à l'unité nationale, de régionalisme et de séparatisme" (HCRA/D, 1998) liés à une mauvaise mise en œuvre de la décentralisation. La question a, par ailleurs, fait l'objet de nombreux débats au sein de la société nigérienne, témoignant les enjeux économiques, sociaux et politiques que recouvre cette réforme.

Dans le cadre de ce premier schéma (1999), une commune était prévue à Bankilaré (en tant que poste administratif), une autre à Yatakala ${ }^{15}$. Les élections locales de février 1999 se sont tenues sur la base des textes de lois de 1998 et de ce schéma. Sur le plan politique, les élections locales se sont soldées par la défaite du parti au pouvoir (le RDP d'Ibrahim Baré Maïnassara), malgré des manœuvres et fraudes électorales, et l'annulation des résultats. Peu après, il s'ensuivait un coup d'Etat militaire et la prise de pouvoir du Commandant Wanké qui assura la transition jusqu'aux élections présidentielles de décembre 1999. Les élections furent remportées par le candidat du MNSD, Tanja Mahamadou. La verne République fut mise en place dans la foulée et le gouvernement, avec à sa tête Hama Amadou (à l'époque Secrétaire général du parti MNSD), prit rapidement fonction au début de l'année 2000.

41 Le projet de décentralisation connut alors une nouvelle période de ralentissement, d'hésitation ou de tiraillements autour de différents schémas de mise en œuvre. La question de la décentralisation fit à nouveau son apparition dans l'ordre du jour de l'Assemblée Nationale fin 2001. Un nouveau schéma fut mis en avant, celui de la communalisation intégrale des cantons et de certains groupements. Ce schéma, dit

Bulletin de I'APAD, 23-24 | 2002 
"schéma 2000", prévoit la création d'environ 200 communes, correspondant approximativement au nombre de cantons existants, plus quelques groupements et les postes administratifs. Des missions visant à informer les leaders politiques, les autorités coutumières (censés répercuter l'information au niveau des populations) et les services déconcentrés de l'Etat ont été menées dans tous les départements du pays.

En avril 2001, sous l'influence d'un ministre originaire du Gorouol, le projet de loi sur la décentralisation fut remanié in extremis pour être validé par le Conseil des Ministres avant d'être transmis à l'Assemblée Nationale.

Ce remaniement avait pour incidence la suppression de la commune de Bankilaré que leur accordait la version précédente du projet de loi.

Disposant de relais au sein du gouvernement, la communauté kel tamasheq de Bankilaré fut aussitôt alertée de la manœuvre. Localement, la réaction fut vive du côté des élites kel tamasheq (leaders politiques, coutumiers et religieux, cadres de l'administration... toutes catégories sociales confondues) qui mobilisèrent leur communauté en organisant diverses manifestations de protestation (marches sur le Poste Administratif, lettre ouverte aux autorités gouvernementales, "sit-in" des 77 chefs de tribus devant l'Assemblée Nationale, menaces de rébellion...). Le cas du Gorouol prenait dès lors les devants de la scène nationale en matière de mise en œuvre de la décentralisation.

Luttes de pouvoir sur fond d'enjeux fonciers et identitaires

Le projet de décentralisation dans sa forme actuelle (communalisation des cantons) propose in fine de superposer au chef de canton ou groupement, sur le même espace territorial, une autorité supérieure ayant les mêmes attributions que le chef coutumier, un maire élu disposant d'une légitimité par les urnes et appuyé par un conseil municipal délibératif au sein duquel la chefferie n'aura a priori, avec 1/5ème des sièges (soit 2 sièges sur 11), que peu d'influence sur les décisions prises. Le projet contient donc plus ou moins en substance une mise à l'écart en douceur des chefs coutumiers du paysage politique local, mise à l'écart dont ceux-ci ont bien pris conscience :

"Nous ne sommes pas contre la décentralisation mais qu'on nous dise clairement quelle sera notre place... ou bien si on veut nous éliminer qu'on le dise clairement !" (un membre du bureau de l'Association des Chefs Traditionnels du Niger, oct. 2001).

A défaut de pouvoir encore lutter contre la réforme au niveau national, les chefs traditionnels l'acceptent mais tentent désormais diverses stratégies individuelles de repositionnement au niveau local.

Du point de vue des chefs coutumiers songhay et kel tamasheq, principaux acteurs de la gouvernance locale dans le Gorouol, l'érection d'une ou bien deux communes rurales est loin d'être un détail. Pour les chefs kel tamasheq, défenseurs de l'option "chacun sa commune", conscients de la difficulté réelle d'amener leurs administrés dispersés sur un très vaste territoire (plusieurs cantons) à voter massivement, le résultat des élections locales sur une seule commune serait favorable aux Songhay qui peuvent plus aisément mobiliser leurs populations regroupées dans une vingtaine de villages. Ainsi, le pouvoir communal leur échapperait totalement.

Quant à la chefferie songhay, partisane de l'option "une unique commune pour un unique territoire", il est clair que la reconnaissance d'une commune aux Kel Tamasheq serait un retour en arrière en termes de reconnaissance de la propriété coutumière des terres, sans compter le surcroît budgétaire que constituerait l'apport des taxes sur les 
populations kel tamasheq pour la future commune du Gorouol (question de viabilité économique).

Aussi, pour justifier son option, l'élite songhay s'appuie-t-elle sur l'existence du décret colonial n 59-127 du 18 juillet 1959, qui créait le Poste Administratif de Bankilaré sans limites territoriales, "dont la compétence s'exerce rationae persone sur les ressortissants des groupements nomades Tinguereguedech-Loghmatten, Doufrafrak et Gaobé". Ce décret est interprété par l'élite songhay comme la preuve "noir sur blanc" de l'absence de légitimité des Kel Tamasheq à réclamer le droit de propriété sur les terres qu'ils occupent. L'argumentaire du Chef de Canton du Gorouol, auquel se rallie solidairement l'ensemble des chefs de canton songhay de l'arrondissement de Téra, et qui correspond plus généralement à la position des chefferies coutumières sédentaires dans les zones agropastorales au Niger, s'appuie sur une interprétation particulière de la communalisation des chefs-lieux de canton.

Du point de vue des chefs de canton songhay, la modalité d'application de la décentralisation accordant une commune aux Kel Tamasheq fixerait définitivement et légalement les populations "nomades" sur des terres ne leur appartenant pas: "Nous n'accepterons pas que nos terres soient divisées et données à d'autres" dit un chef de canton de la zone à propos d'un cas similaire (cas des peuls de Diagourou qui seraient installés sur les terres des Songhay de Téra). Il y a là un peu de revanche historique sur les "nomades" en termes de domination et de luttes de pouvoir.

Dans cette zone à l'extrême Ouest du Niger, les disputes entre Songhay et Kel Tamasheq sur les dégâts champêtres, l'accès aux points d'eau et le non-respect des couloirs de passage, sont fréquentes et anciennes (cela est vrai pour toutes les zones agropastorales où les activités d'élevage et d'agriculture sont en situation de forte concurrence). Plusieurs conflits violents se sont traduits par des morts d'hommes, voire des massacres.

Par simple effet d'annonce, le projet de mise en œuvre de la décentralisation relance les enjeux fonciers, ravive les tensions et exacerbe les positions des antagonistes. Cependant il ne convient pas d'opposer les agriculteurs sédentaires songhay d'une part et les éleveurs nomades kel tamasheq d'autre part, puisque les uns et les autres sont aujourd'hui tous sédentaires et pratiquent à la fois l'élevage et la culture vivrière. Un repli identitaire semble néanmoins s'opérer derrière l'ethnie, et plus loin la dichotomie sédentaires/nomades refait surface. Les dissensions internes de chaque communauté sont tues, tandis que les discours sur l'autre oscillent entre la cohabitation harmonieuse des modes de production agricole et pastorale et le règlement par la force des conflits d'intérêt. Ce qui est au cœur de cette dispute jusque là verbale, quoique épisodiquement violente, c'est en particulier la propriété et le droit d'usage de la terre des populations pastorales.

53 Le Code Rural nigérien statue sur cette question centrale de la propriété de la manière suivante (ordonnance 93-015, article 9) :

"La propriété coutumière résulte de l'acquisition de la propriété foncière rurale par succession depuis des temps immémoriaux et confirmée par la mémoire collective ou par l'attribution à titre définitif de la terre à une personne par l'autorité coutumière compétente."

54 La loi ne fait qu'entériner des pratiques foncières mais ne résout rien. Aussi, la revendication pour la propriété de la terre prend évidemment l'allure de bataille de légitimation historique, où la démonstration de la préséance sur la terre est centrale. 
Dans ces joutes, jusque là discursives, la manipulation de l'histoire est dès lors devenue un exercice auquel se livre chacun des deux camps, jouant sur l'étymologie des noms de lieux, sur les types de relations qui prévalaient entre les deux groupes (accueil, cohabitation, allégeance), etc. Mais, comme le souligne Bernus, "chaque groupe humain construit sa propre vérité à laquelle il croît et essaie de l'imposer aux autres" (Bernus 1990).

Sans prétendre relater ici de manière approfondie l'histoire du peuplement de la zone ${ }^{16} \mathrm{ni}$ arbitrer ce débat local, il n'est pas inutile de souligner que, comme souvent ailleurs en Afrique de l'Ouest, les populations actuelles du Gurma (kel tamasheq, gaobe et songhay) se sont en fait progressivement installées au cours des siècles dans un domaine qui n'était pas le leur et où elles se sont infiltrées par vagues migratrices ou imposées par la force, les rapports de force ayant été en perpétuelle évolution. Au moment de la pénétration coloniale, il apparaît que la zone était dominée par les Kel Tamasheq. Ceux-ci furent eux-mêmes soumis à la domination militaire française qui rapidement entreprit d'asseoir son administration en s'appuyant sur les chefferies. En 1927, un décret délimitait les cantons des Songhay et celui des Loghmatten, mais ce dernier par la suite disparaissait. En 1955, un arrêté du Gouverneur du Niger définissant les domaines de compétences des chefs de canton et de groupement précisait que les premiers ont une autorité sur un territoire délimité contrairement aux seconds dont l'autorité s'exerce sur les personnes qui leur sont rattachées. Ainsi, cette distinction entre nomades et sédentaires était déjà largement orientée par les textes coloniaux.

Mais la bataille s'engage aussi sur d'autres terrains avec d'autres armes. Les Songhay, forts de leur groupe de pression (association de ressortissants basée à Niamey) et de leur "ministre-cartouche" ${ }^{17}$, tentent d'imposer leur point de vue en œuvrant dans les plus hautes instances de l'appareil d'Etat.

Quant aux Kel Tamasheq, dont la nombreuse élite locale (77 chefs de tribus), unie solidairement derrière son chef de groupement, est consciente de sa faiblesse relative liée au manque de relais au niveau national (y compris auprès des ex-rebelles intégrés au gouvernement), ils multiplient les manifestations diverses tout en faisant valoir l'argument de certains dirigeants de la Rébellion relatif à l'administration des groupes minoritaires. Du même coup ils tentent de bénéficier du ralliement à un drapeau communautaire kel tamasheq qui a valu des positions de pouvoirs à quelques uns des "nordistes" ${ }^{18}$ et menacent d'une nouvelle Rébellion (dont ils n'ont guère les moyens).

"Notre terre s'arrête là où commence celle des bella", répond un chef de village songhay du Gorouol à la question de la délimitation territoriale du village. Cette phrase semble bien indiquer que le débat actuel sur la propriété foncière des Kel Tamasheq de cette zone est plus la lutte d'une élite, en situation de perte de pouvoir et menacée par le projet de décentralisation, qu'un problème de fond exprimé par la base, tel que le présente le chef de canton du Gorouol :

"Les gens ne vont jamais accepter qu'on divise leurs terres pour attribuer une partie à d'autres personnes, vous voyez que ce n'est pas faisable, et c'est ce que j'ai refusé d'accepter."

De l'autre côté, les iklan qui forment la grande majorité des populations kel tamasheq de Bankilaré sont également peu informés des enjeux réels de la communalisation. Largement manipulés, ils répondent présents à l'appel pour la mobilisation lancé par la hiérarchie qui, jalouse de ses pouvoirs et prérogatives, aussi maigres soient-elles, se garde bien de révéler les véritables enjeux de la décentralisation et agite éperdument le 
drapeau identitaire et la nécessaire unité face aux menaces d'exclusion (menaces réelles sur une question cruciale) dans l'accès à la propriété foncière.

La question identitaire évoquée précédemment n'est qu'un des aspects du problème et je ne $\mathrm{m}^{\prime} \mathrm{y}$ attarderai pas plus si ce n'est en mentionnant que le Recensement Général de la Population (RGP) réalisé en 2001 fournit un bon exemple de ce mouvement de repli et une nouvelle occasion de réaffirmer des identités communautaires distinctes.

"Lors du recensement, il y a eu ce problème : imprimé "Canton de Gorouol." Les buzu ${ }^{19}$ ont dit "non" de se faire recenser sur ces imprimés, sauf si on écrit "groupement." Ils ont raison! Les imprimés dans le temps c'était "village ou tribu." Ils ont bloqué le RGP pendant 72 heures! Il a fallu que le Sous-Préfet vienne de Téra. S'ils se font recenser sous Gorouol, alors ils n'existent plus! C'est pourquoi la question de la commune revient ici," (un gendarme hausa basé à Bankilaré).

Cette instrumentalisation d'un problème toutefois réel (si l'on en juge par la fréquence des conflits fonciers) a eu pour effet de regrouper les individus derrière la bannière ethnique, mettant de côté toutes les dissensions internes qui animent de part et d'autre les communautés. Ainsi, au-delà des conflits familiaux qui ont toujours animé la vie de la chefferie songhay, au-delà des disputes politiques qui depuis les indépendances ont divisés les individus d'un même village, d'un même campement, au-delà du combat de fond mené par les iklan pour gagner leur autonomie vis-à-vis de la noblesse ou des groupes maraboutiques, face à l'enjeu foncier les rangs se sont resserrés pour constituer deux camps radicalement opposés.

En guise de conclusion

Le projet de décentralisation au Niger est d'ores et déjà perçu comme un retrait de l'Etat qui semble laisser la porte ouverte à diverses formes d'appropriation ou de dérives. Si ces dernières sont bien perçues par l'Etat en tant que risques politiques liés à la mise en œuvre du projet, elles n'ont pas trouvé de parade sur le plan législatif. Dans leur état actuel, les lois sur la décentralisation sont très défavorables aux nomades, en particulier dans les zones où ils sont en situation de minorité. Tandis qu'au niveau national, la création des circonscriptions électorales spéciales avait permis de garantir le droit des minorités à une représentation au niveau de l'Assemblée Nationale du Niger, rien n'est prévu au niveau décentralisé.

Et, déjà, on recense des projets d'école ou de puits qui ont été refusés par la chefferie songhay à des "nomades" (qui n'en sont plus!) du Gorouol, sous prétexte du risque d'appropriation des terres.

64 L'exclusion a priori de certains acteurs est un premier élément de constat auquel les autorités qui mettent en œuvre la décentralisation auront à faire face rapidement. Contrairement aux principes généraux qui engagent le gouvernement et ses partenaires du Nord a accélérer ce processus, de telles perspectives ne présagent guère la "bonne gouvernance" attendue.

Ceci est d'autant plus à craindre que l'on constate qu'en fait de gouvernance, la gestion des affaires publiques semble être un laissé pour compte du développement. Face à des logiques d'appropriation et d'accaparement des biens collectifs, dans des situations de concurrence pour le pouvoir et le contrôle des ressources (naturelles, humaines et financières), et à des pratiques clientélistes et corruptrices, la "main invisible" d'Adam Smith ne fonctionne pas tel que l'entendent les initiateurs du projet de décentralisation. 
66 des élites locales, s'avère soit fictive soit embryonnaire et muselée et, en tout état de
cause, n'a guère d'emprise sur les réalités politiques locales, si bien qu'il est à craindre
que les objectifs participatifs de la décentralisation soient mis à mal et largement
détournés.

\section{BIBLIOGRAPHIE}

Bailey F.-G., 1969/1971, Les règles du jeu politique, Paris : PUF.

Bernus E., 1974, "L'évolution récente des relations entre éleveurs et agriculteurs en Afrique Tropicale : l'exemple du Sahel nigérien", Cahiers de l'ORSTOM, Sciences humaines, vol. XI, n² : 137-143.

Bernus E., 1990, "Histoires parallèles et croisées : nobles et religieux chez les touaregs Kel Denneg", L'Homme, 115, juil-sept. Vol. XXX, n³ : 31-47.

Bierschenk T., Chauveau J.-P., Olivier de Sardan J.-P. (dir), 2000, Courtiers en développement. Les villages africains en quête de projets, Paris, APAD - Karthala.

Bierschenk T., Olivier de Sardan, J.-P. (eds), 1998, "Les arènes locales face à la décentralisation et démocratisation. Analyses comparatives en milieu rural béninois", in Bierschenk T. \& Olivier de Sardan J.-P. (eds), Les pouvoirs au village. Le Bénin rural entre démocratisation et décentralisation, Paris, Karthala : 11-51.

Bourgeat A., 1995, Les sociétés touarègues : nomadisme, identité, résistances, Paris, Karthala.

Bourgeat A., 1999, Horizons nomades en Afrique sahélienne. Sociétés, développement et démocratie, Paris : Karthala.

Cercle de Téra, 1960, Bulletin mensuel de renseignement.

Cercle de Téra, 1963, Rapport annuel.

Evers H.D. \& Schiel T., 1988, Strategische Gruppin. Vergleichende Studien zu Staat, Bürokratie und Klassenbildung in der Dritten Welt, Berlin : Reimer.

Gallais J., 1975, Pasteurs et paysans du Gourma. La condition sahélienne, Bordeaux ; Centre d'études de géographie tropicale du CNRS.

Giraut F., 1999, Retour du refoulé et effet chef-lieu. Analyse d'une refonte politico-administrative virtuelle au Niger, Grafigéo, $\mathrm{n}^{\circ}$ 7, PRODIG.

Grégoire E., 1999, Touaregs du Niger, le destin d'un mythe, Paris ; Karthala.

HCRA/D, "Les risques de résurgence de féodalités locales, d'aggravation de déséquilibres régionaux, de mauvaise gestion, d'atteinte à l'unité nationale, de régionalisme et de séparatisme", 1998.

Moore S.F., 1978, Law as process, London : Routledge et Kegan Paul. 
Sere de Rivières E. , 1965, Histoire du Niger, Collection Mondes d'Outre mer, Paris : Editions Berger-Levrault.

Tidjani Alou M., 2000, "Démocratie, exclusion sociale et quête de citoyenneté ; cas de l'association Timidria au Niger", Journal des Africanistes, vol. 70, n 1-2 ; 173-195.

\section{NOTES}

1.J'utiliserai ici plus volontiers le terme kel tamasheq (dans lequel les individus appartenant à ce groupe se reconnaissent) que les termes utilisés par les groupes voisins (surgey, belley, etc.) ou encore le terme français "touareg", aujourd'hui contesté par certains acteurs (notamment l'association de lutte contre l'esclavage Timidria). Le terme désigne l'ensemble des individus de langue et de culture tamasheq.

2.Il faut néanmoins noter que des liens des acteurs locaux avec des acteurs basés à l'étranger (Côte d'Ivoire, Arabie Saoudite) viennent complexifier davantage les jeux stratégiques au niveau local.

3. "Groupes stratégiques" est entendu dans le sens défini par Bierschenk et Olivier de Sardan (1998 : 262-265), se référant à Evers (Evers et SchieI 1988), c'est-à-dire en tant qu'agrégats sociaux, à géométrie variable, qui défendent des intérêts communs, en particulier par le biais de l'action sociale et politique. Notre étude ne s'est pas pour autant limitée à ces catégories.

4.Malgré son abolition officielle, l'esclavage reste une forme de rapports sociaux qui s'est maintenue dans certaines zones du Niger.

5.Le dernier exemple remonte à la campagne hivernale 2001, où un cultivateur songhay ayant mis en culture un espace qu'il estimait en friche, s'est vu opposer une résistance armée des Kel Tamasheq voisins qui revendiquaient l'appartenance de cette terre et sa mise en réserve pour des usages pastoraux (source : entretiens et éléments du dossier passé en justice, sept. 2001).

6.Les Gaohé ont d'eux mêmes cherché à prendre de la distance vis-à-vis de Bankilaré qu'ils considéraient comme "capitale" de leurs anciens ennemis Tinguereguedech (archives de Téra, 1960).

7.Les très nombreuses tribus vassales soumises à "autorité de l'amenokal des Kel Igirer ont conquis peu à peu, avec par intermittence l'encouragement de l'administration française, une autonomie et une indépendance économique vis-à-vis de leurs anciens maîtres (appropriation du bétail, propriété privée des produits de leurs champ., de cultures, propriété de leur descendance, non versements des tributs...). Bien que le mouvement d'émancipation soit très avancé, on doit encore parler aujourd'hui de processus en cours. Un tel mouvement est soutenu par une organisation nationale (association Timidria) de lutte contre l'esclavage (Tidjani Alou 2000).

8.Les chefs de canton et chefs de groupement sont rémunérés par une allocation annuelle octroyée par l'administration "en fonction de l'importance démographique et politique" de la chefferie considérée (article 12, arrêté n 2566/APA du 16 novembre 1955).

9.Le groupe maraboutique est formé de plusieurs familles pour la plupart originaires de Esuk, réparties dans différentes tribus. Certains ont acquis une grande influence dans les prises de décision qui concernent l'ensemble de la Confédération. A l'origine, ces différentes familles ne seraient pas des Kel Tamasheq mais des arabes instruits du Coran (sheriffen) devenus les marabouts des KeI Tamasheq, les assistants dans leurs 
combats par les prières et les talismans qu'ils confectionnent. Ils cumulent souvent les fonctions de chefs religieux et de chef de tribus. Ils concluent fréquemment des alliances matrimoniales avec les imajeghen Tinguereguedech-Loghmatten. Ils assurent également le règlement de toutes les affaires religieuses et matrimoniales (mariages, séparations, divorces, héritages.., ).

10.Sur la notion de "courtiers en développement" voir Bierschenk, Chauveau, Olivier de Sardan, 2000.

11.Littéralement "Ceux de la vallée".

12.Aucun front ne fut formé dans le Gurma, mais quelques jeunes s'engagèrent ici et là dans différents fronts armés au Mali (FPLA), dans l'Aïr (FPLS) ou tentèrent l'aventure en Algérie et en Lybie.

13.Pour une explication de la genèse du conflit touareg, voir Grégoire (1999 : 57-71).

14.Il convient de préciser ici que les communes se superposent aux cantons et groupements, dont la compétence territoriale est souvent plus large.

15.L'érection du village de Yatakala, à l'époque chef-lieu de Canton du Gorouol, en chef-lieu de commune fut consentie par le gouvernement pour calmer les revendications territoriales des Songhay.

16.Sur ce point, pour des éléments détaillés, voir Séré de Rivières (1965).

17.Pour reprendre l'expression imagée d'un membre de la chefferie Tinguereguedech-Loghmatten, en référence aux cartouches des rebelles qui rétablirent quelque peu la minorité kel tamasheq sur l'échiquier national.

18.Les ex-rebelles intégrés sont des anciens chefs de fractions de la Rébellion, originaires de l'Aïr et de l'Azawagh, peu sensibles à la situation de ceux du Gurma. 19."Huzu" désigne en hausa les captifs kel tamasheq, mais ce terme est plus souvent employé pour désigner l'ensemble des Kel Tamasheq, au même titre que les Songhay et Zarma utilisent le terme "bella".

\section{AUTEUR}

\section{ERIC KOMLAVI HAHONOU}

Doctorant en anthropologie à l'EHESS Marseille. chercheur au LAS DEL BP 12901

Niamey. Tél. : (227) 72.37.80 ; komlavi@intnet.ne 